\title{
Folgen insuffizienter Ernährung
}

Verschiedene klinische Studien haben gezeigt, daß durch ausschließliche parenterale Zufuhr optimal zusammenge-setzter Nährlösungen ein ungestörter Stoffwechsel über viele Monate aufrechterhalten werden kann. Durch den enormen Fortschritt auf dem Gebiet der langfristigen parenteralen Emährung können heute Menschen gerettet werden, die früher in der Katabolie verstarben. Der Nutzen kurzfristiger intravenöser Emährung wird dagegen von vielen Klinikem weit weniger gewürdigt, ja sogar teilweise in Frage gestellt.

Die Pathophysiologie des Hungerstoffwechsels zeigt, daß ein ständiger Substratfluß durch den Organismus Voraus-setzung für eine optimale Aufrechterhaltung der Homöo-stase ist. Unterbrechung der Nahrungszufuhr führt zu Ver-änderungen des Stoffwechsels und zu einer Einschränkung derHomöostase. Schonnach 12-16 Stunden sind die Glyko-genspeicher des Organismus erschöpft und können danach nur durch Glukoneogenese aus Körperprotein aufgefüllt werden. Um den Tagesbedarf des Gehims von 100-140 g Glukose ausreichend zu decken, müssen $250 \mathrm{~g}$ Eiweiß abge-baut werden. Bis in die letzten Jahre hat man fälschlicherweise angenommen, diese bei Nahrungskarenz auftreten-den Stickstoffverluste gingen ausschließlich zu Lasten der quergestreiften Muskulatur. Es war üblich geworden, die Stickstoffverluste in g Muskelgewebe (N x 6,25 x 5) umzu-rechnen und deshalb die negative Stickstoffbilanz insge-samt für unbedeutend zu halten. Гnzwischen haben Befun-de von Ghadimi, Niklas und Waterlow bewiesen, daß im Hunger zunächst die schnell verfügbaren Proteine mit kurzer Halbwertszeit mobilisiert werden. Dazu gehören nach Tabelle I neben den wichtigen Enzymproteinen von Leber, Pankreas und Darmmukosa u. a. auch die Proteine der Tubulusepithelien der Nieren. Bei Mangelernährung muß aufgrund dieser Daten mit einer frühzeitigen Einschränkung der Leistungsbreite der betroffenen Organe gerechnet werden. Im Bereich des Gastrointestinaltraktes und der damit verbundenen Verdauungsdrüsen bedeutet das eine mangelhafte Verwertung oral angebotenen Proteins. Entsprechend Abbildung 1 kann sich ein Circulus vitiosus entwickeln, der häufig allein durch parenterale Emährung zu unterbrechen ist [GhadimÍJ.

Fasten oder mangelnde Prate inzufuhr

Abbau von Muskelproteinen (Halbwertszeit: mehrere Wch.)

$1 / 8$ Eiweißmangel

Unterbrechung durch geeignete therapeutische Maßnahmen, z.B. parenterale Emährung $1 / 2 Z$

Unfáhigkeit

oral angebotene Substrate

zu verwerte $\pi$

Abbau von Enzymen des G.I.--Traktes und der Leber (Halbwertszeit: einige Std.)

Ungeeignete therapeutische

Maßnahmen: konventionelle Emährung 
Abbildung 1. Induktion eines Circulus vitiosus im Verdauungs-trakt durch ungenügende Eiweißversorgung [nach Coats].

Tabelle I. Absolute mittlere Lebensdauer des Eiweißes verschie-dener Gewebe. Innerhalb der angegebenen Zeiten wird das ganze Zelleiweiß dem Betrag nach einmal umgebaut [nach Niklas]. Gewebe

Mittlere Lebensdauer

Exokrine Pankreasepithelien 6 Stunden

Hauptzellen des Drüsenmagens Epithelien seröser Speicheldrüsen

RES in Leber, Milz, Lymphknoten 9 Stunden

Leberepithelien 14 Stunden

Tubulusepithelien der Niere

Skelettmuskulatur 32 Tage

Die Bedeutung adäquater Emährung geht auch aus den Untersuchungen von Wuhrmann und Märki eindruck-voll hervor. Sie bewiesen eine direkte Abhängigkeit von Serum-Eiweiß- bzw. Albuminspiegeln und Mortalitätsraten bei internistischen Patienten. Wie Tabelle //zeigt, steigt die Mortalität bei einem Absinken des Albumins unter 3,0 g\% auf das 5 fache an. Kudlicka et al. konnten unter Appli-kation einer Standarddiät durch zusätzliche Verabreichung von $500 \mathrm{ml}$ Aminofusin ${ }^{\circledR} L$ forte an jedem zweiten Tag neben anderen positiven Effekten eine Neubildung von durchschnittlich 1-3 g Albumin pro Tag erzielen. (Abbildung 2).

Nach Gofferje darf aufgrund der kurzen Halbwertszeit der Proteine der Tubulusepithelien der Nieren eine früh-zeitige Applikation von Aminosäuren bei Patienten mit akutem Nierenversagen nicht länger als Kontraindikation angesehen werden. Abel el al. konnten durch frühzeitige intravenöse Applikation essentieller Aminosäuren und ausreichender Mengen von Energieträgern die Letalität des akuten Nierenversagens von $56 \%$ einer isokalorisch, aber aminosäurenfrei ernährten Kontrollgruppe auf 25\% senken.

Aufgrund dieser Ergebnisse und im Hinblick auf die neuen heute zur Verfügung stehenden Nährlösungen (Tabelle III) und die moderne Infusionstechnik sollte auch im inter-nistischen Bereich keinem Patienten eine adäquate Sub-stratzufuhr vorenthalten werden. Tee und Haferschleim können eine parenterale Ernährung nicht ersetzen.

Tabelle II. Die Abhängigkeit des Serum-Eiweiß- bzw. Albumin-spiegels von Mortalitätsraten internistischer Patienten [Wuhr-mann und Märki].

Abbildung 2. Änderung von klinischen Parametern nach Zufuhr von 9 Infusionen Aminofusin ${ }^{\circledR}$ L forte in 16 Tagen.

$\pi$

Literatur

Abel, R. M.; Beck, D. H.; Abbott, W. M.; Ryan, J. A.; Barnett, G. O., and Fischer, J. E.: Improved survival from acute renal failure after treatment with intravenous essential 1 -amino acids and glucose. New Engl. J. Med. 288:695 (1973). Coats, D. A.: Langfristige parenterale Ernährung bis zu 7 Monaten Dauer. Erfahrungen in Australien. Langenbecks Arch. chir. Suppl. chir. Forum 7972:175. Ghadimi, H.; Kumar, S., and Abaci, F.: Endogenous Amino 
Acid. Loss and its Significance in Infantile Diarrhea. Pediat.

Res. 7:161 (1973). Gofferje, H.: Grundlagen der parenteralen Ernährung des akuten

Nierenversagens. Infusionstherapie 7:366 (1973/74). Kudlicka, V.; Doberský, P.; Vulterinová, M. und

Kudlicková V.: Die Auswertung der Aminosäurenbehand-

lung auf Grund von Messungen der Albuminindistribution;

in Doberský Rehabilitation durch Ernährung. Z. Ernähr.-Wiss.

Suppl. 75:53(1972). Nicklas, A.; Quincke, E.; Maurer, W. und Neyen, H.: Biochem.

Z. 7:330(1958). Waterlow, J. C. and Stephen, J. M. L.: The assessment of protein nutrition and metabolism in the whole animal with special reference to man; in Munro, Mammalian protein metabolism,

vol. 3, p. 325 (Academic Press, New York 1969). Wilmore, D. W. and Dudrick, S. J.: Treatment of acute renal

failure with intravenous essential L-amino acids. Arch. Surg.

99:669 (1969). Wuhrmann, F. und Märki, H. H.: In Dysproteinämien und Para-

proteinamien: Grundlagen, Klinik und Therapie (Schwabe,

Basel/Stuttgart 1963).

Tabelle III. Infusionsplan. $2500 \mathrm{ml}$ Flüssigkeit 2100 Kalorien, $50 \mathrm{~g}$ Aminosäuren, $460 \mathrm{~g}$ Kohlenhydrate. Infusion über periphere Ve-nen, langfristig über einen Cava-Katheter.

die richtige

pfrimmer lösung 